\title{
On the determination of elastic coefficients from indentation experiments
}

\author{
$\mathrm{N}$ Tardieu and A Constantinescu \\ Laboratoire de Mécanique des Solides, (CNRS UMR 7649, Polytechnique, Mines, Ponts and \\ Chaussées), 91128 Palaiseau cedex, France
}

\begin{abstract}
The main result of this paper is the extension of the adjoint state method to variational inequalities. This is done for the Signorini contact problem (Kikuchi N and Oden J T 1988 Contact Problems in Elasticity: a Study of Variational Inequalities and Finite Element Methods (Philadelphia: SIAM)) and used for the identification of elastic coefficients from an indentation test. The result is obtained by two independent approaches based on the penalized and respectively, mixed formulations of the direct problem, a Signorini contact problem. An important and astonishing result is that the obtained adjoint problem is a linear problem with Dirichlet boundary conditions. This is expected for problems described with variational equalities (Bui H D 1993 Introduction Aux Problèmes Inverses en Mécanique des Matériaux (Paris: Eyrolles) (Engl. Transl. (Boca Raton, FL: CRC Press)), Lions J L 1968 Contrôle Optimal des Systèmes Gouvernés par des Équations aux Dérivées Partielles (Dunod)), but is a new result for problems described with variational inequalities. As an application, the elastic coefficients of an isotropic body have been identified from the knowledge of a displacement-force curve measured during an indentation test. The efficiency of the method is illustrated on numerical examples for the identification of a bimaterial and a functional gradient material.
\end{abstract}

\section{Introduction}

The indentation test consists in pressing a punch of arbitrary shape (conical, spherical, pyramidal, etc) into a material sample (figure 2) and recording the penetration depth versus the reaction force (figure 1). The underlying identification problem seeks to recover the material coefficients of the constitutive law from the knowledge of an indentation test result. The importance of this testing method stems from its experimental simplicity. However, the interpretation of the test results is not complete in spite of the large amount of interest shown by the engineering community over the last decades.

From a mathematical point of view, the direct indentation problem of a linear elastic material corresponds to an elliptic partial differential equation with unilateral constraints. The variational formulation of this problem therefore conducts to the use of variational inequalities with variables in closed convex sets [15]. The associated inverse problems inherit this mathematical complexity.

The existing solutions to the identification problem of the material parameters (i.e. coefficients of the associated partial differential equation) from indentation tests are on the one hand semi-empirical formulae in some particular cases of material behaviour $[5,8,13,14,18]$. On the other hand, one finds attempts to solve a related minimization problem for a given cost functional without any reference to a gradient computation $[10,11,16]$. The minimization 


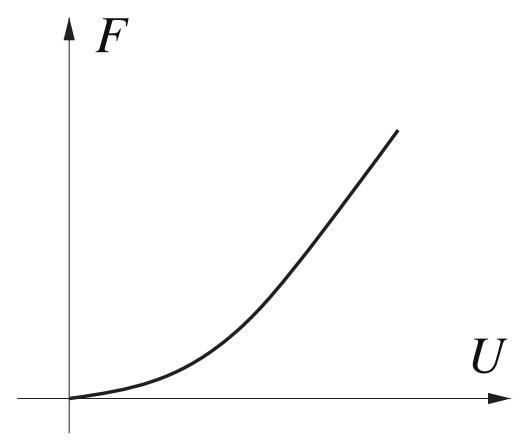

Figure 1. Indentation curve.

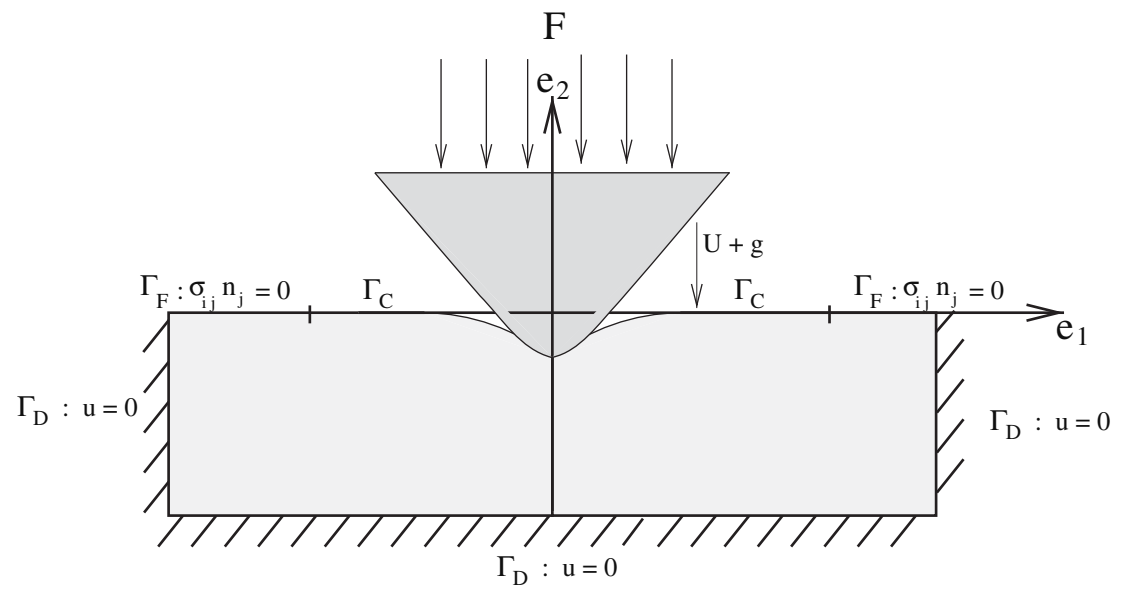

Figure 2. Indentation test (direct problem $(\mathcal{P})$ ).

approach is obviously of primal interest and would be enhanced by the possibility to compute the gradient of the cost functional with respect to the material parameters.

The purpose of this paper is to show that the gradient of the cost functional can be determined for an inverse elastic indentation problem. With this aim, the adjoint state method has been modified to apply to variational inequalities. As a consequence, an exact gradient is obtained from the knowledge of the solutions to the direct and the adjoint problems. The adjoint problem has been deduced using two different approaches based on the penalized and respectively, mixed variational formulations of the Signorini contact problem. An important and astonishing result is that the obtained adjoint problem is a linear problem with Dirichlet boundary conditions. This is expected for problems described with variational equalities [2,17], but is a new result for problems described with variational inequalities.

In section 2, the direct contact problem is presented using three different weak formulations [15]: primal, penalized and mixed. In section 3, the inverse problem is expressed as a constrained minimization problem.

In section 4, the application of the adjoint state method for variational inequalities is presented. The direct and adjoint problems are obtained as sufficient conditions for the stationarity of a Lagrangian functional.

Finally, a series of numerical examples illustrates the efficiency of the method by identifying coated and functional gradient materials from an indentation test with a conical punch. 


\section{The direct contact problem}

Let us consider an axisymmetric body, with its section occupying in its reference configuration an open subset $\Omega \subset \mathbb{R}^{2}$ with smooth boundary $\Gamma$ (figure 2). The boundary is partitioned in three parts $\Gamma=\Gamma_{D} \cup \Gamma_{F} \cup \Gamma_{C}$ : the part $\Gamma_{D}$ where displacements are imposed, the free surface $\Gamma_{F}$ and the surface $\Gamma_{C}$ where contact might occur. We shall denote by $\Gamma_{C}^{\mathrm{eff}} \subset \Gamma_{C}$ the part where contact is effective.

The problem considered in what follows is the indentation of the body $\Omega$ by a rigid punch, whose profile is characterized by the gap $g$ (the distance between the punch and the surface $\left.\Gamma_{C}\right)$. The contact is considered without friction. One can note that the effective contact region $\Gamma_{C}^{\mathrm{eff}}$ is not known in advance, causing the nonlinearity of the direct problem.

In order to simplify the presentation without restricting the generality of the method, the configuration is supposed axisymmetric and no body forces or imposed surface tractions are considered.

The real and the virtual displacement fields $\boldsymbol{u}, \boldsymbol{v}$ belong to the following functional space:

$$
\boldsymbol{V}=\left\{\boldsymbol{v} \in\left(H^{1}(\Omega)\right)^{2} \mid \boldsymbol{u}=\mathbf{0} \text { on } \Gamma_{D}\right\} .
$$

Under the assumptions of small strains and an elastic constitutive law, the displacement vector, the second-order strain and stress tensors are subject to the following set of equations:

$$
\begin{aligned}
& \boldsymbol{\varepsilon}(\boldsymbol{u})=\frac{1}{2}\left(\nabla \boldsymbol{u}+\nabla^{T} \boldsymbol{u}\right) \\
& \boldsymbol{\sigma}(\boldsymbol{u})=\boldsymbol{A}(\boldsymbol{c}): \varepsilon(\boldsymbol{u})
\end{aligned}
$$

where $\boldsymbol{A}(\boldsymbol{c})$ is the fourth-order tensor of elastic moduli depending of a vector of material parameters $c \in \boldsymbol{L}$, a closed subset of $\mathbb{R}^{n}(n \geqslant 1)$. The stress tensor $\boldsymbol{\sigma}$ also satisfies the equilibrium equations:

$$
\operatorname{div} \sigma(\boldsymbol{u})=\mathbf{0} .
$$

Using the previous notations, we will recall three classical variational formulations of the frictionless contact problem between a rigid punch of arbitrary shape and an elastic foundation, denoted as the Signorini problem (for a detailed presentation see Kikuchi and Oden [15]).

\subsection{Primal formulation $(\mathcal{P})$}

This problem is driven by the indentation depth $U^{\exp }$ and is written as follows:

find $\boldsymbol{u} \in \boldsymbol{K}$ such that

$$
\begin{aligned}
& \int_{\Omega} \boldsymbol{\sigma}(\boldsymbol{u}): \boldsymbol{\varepsilon}(\boldsymbol{v}-\boldsymbol{u}) \mathrm{d} \Omega \geqslant 0 \\
& \forall \boldsymbol{v} \in \boldsymbol{K}=\left\{\boldsymbol{v} \in \boldsymbol{V} \mid v_{2} \leqslant g+U^{\exp } \text { on } \Gamma_{C}\right\} .
\end{aligned}
$$

The resultant force $F$ on the indenter is computed from the solution $u$ of the contact problem by

$$
F=\int_{\Gamma_{C}} \sigma_{22}(\boldsymbol{u}) \mathrm{d} \Gamma
$$

Within this formulation, the contact conditions are directly imposed to the solution $\boldsymbol{u}$ by searching it in the closed convex set $\boldsymbol{K}$. This causes a certain number of difficulties from a numerical point of view and therefore equivalent formulations on vector spaces are to be preferred. 


\subsection{Penalized formulation $\left(\mathcal{P}_{\epsilon}\right)$}

For an arbitrary small $\epsilon>0$, we consider the following problem:

find $\boldsymbol{u}_{\epsilon} \in \boldsymbol{V}$ such that

$$
\int_{\Omega} \boldsymbol{\sigma}\left(\boldsymbol{u}_{\epsilon}\right): \boldsymbol{\varepsilon}(\boldsymbol{v}) \mathrm{d} \Omega+\frac{1}{2 \epsilon} \int_{\Gamma_{C}}\left\langle u_{\epsilon 2}-g-U^{\exp }\right\rangle_{+}^{2} v_{2} \mathrm{~d} \Gamma=0 \quad \forall \boldsymbol{v} \in \boldsymbol{V}
$$

where $\langle\cdot\rangle_{+}$denotes the positive part.

The reaction force $F_{\epsilon}$ on the punch is defined as

$$
F_{\epsilon}=-\frac{1}{2 \epsilon} \int_{\Gamma_{C}}\left\langle u_{\epsilon 2}-U^{\exp }-g\right\rangle_{+}^{2} \mathrm{~d} \Gamma
$$

In this case, one can consider in a rough interpretation that the contact conditions are replaced by a nonlinear contact force proportional to the allowed interpenetration and inverse proportional to $\epsilon$. Some convergence properties are proven: $\boldsymbol{u}_{\epsilon}$ converges strongly to $\boldsymbol{u}$ in $\boldsymbol{V}$ and $F_{\epsilon}$ converges to $F$ in $\mathbb{R}$. Formally, this means that, as $\epsilon \rightarrow 0$, the solution to the penalized problem $\left(\mathcal{P}_{\epsilon}\right)$ converges to the solution to the primal problem $(\mathcal{P})$.

\subsection{Mixed formulation $\left(\mathcal{P}_{m}\right)$}

This formulation has the following expression:

$$
\begin{aligned}
& \text { find }(\boldsymbol{u}, p) \in \boldsymbol{V} \times \boldsymbol{N} \text { such that } \\
& \qquad \begin{array}{l}
\int_{\Omega} \boldsymbol{\sigma}(\boldsymbol{u}): \boldsymbol{\epsilon}(\boldsymbol{v}) \mathrm{d} \Omega-\int_{\Gamma_{C}} p \cdot v_{2} \mathrm{~d} \Gamma=0 \quad \forall \boldsymbol{v} \in \boldsymbol{V} \\
\int_{\Gamma_{C}}(q-p) \cdot\left(u_{2}-U-g\right) \mathrm{d} \Gamma \geqslant 0 \quad \forall q \in \boldsymbol{N} .
\end{array}
\end{aligned}
$$

The contact condition have been relaxed in this case by the introduction of the Lagrange multiplier $p$. The displacement field $\boldsymbol{u}$ is now supposed to belong to the vector space $\boldsymbol{V}$ and the Lagrange multipliers $p$ to the convex cone $N=\left\{q \in\left(H^{1 / 2}\left(\Gamma_{C}\right)\right)^{\prime} \mid q \leqslant 0\right\}$, where (.) denotes the dual of (.).

The Lagrange multiplier $p \in N$ shows up to be the pressure distribution under the punch. Therefore, the resultant force $F$ is expressed as

$$
F=\int_{\Gamma_{C}} p \mathrm{~d} \Gamma
$$

\section{The inverse problem}

The inverse problem addressed here is the identification of the material properties from a displacement-force measurement curve on the indenter. From a practical point of view, it is interesting to consider the inverse problem as a minimization problem of the following form $[9,16]$ :

find $c^{*} \in \boldsymbol{L}$ such that

$$
\mathcal{J}\left(\boldsymbol{c}^{*}\right)=\min _{\boldsymbol{c} \in \boldsymbol{L}} \mathcal{J}(\boldsymbol{c})
$$

where $\mathcal{J}$ represents a cost functional involving measured and computed quantities. Different cost functionals can be imagined:

- the difference between measured and computed forces $F^{\exp }, F$ for given material parameter $c$ and a prescribed indentation depth $U^{\exp }$ :

$$
\mathcal{J}(c)=\frac{1}{2}\left(F-F^{\exp }\right)^{2}
$$


- the difference between measured and computed displacements $U^{\exp }, U$ for imposed indentation force $F^{\exp }$ :

$$
\mathcal{J}(c)=\frac{1}{2}\left(U-U^{\exp }\right)^{2}
$$

- the reciprocity gap, i.e., the crossed difference between computed and measured forces and displacements:

$$
\mathcal{J}(c)=\left(U^{\exp }-U\right)\left(F-F^{\text {exp }}\right) .
$$

If the preceding cost functionals have been defined for a single definite indentation depth, one can also imagine cost functionals as sums or time integrals of these quantities. In this paper, we shall consider simple cost functionals based on the squared difference between computed and experimental forces (12).

\section{The adjoint state method for variational inequalities}

In the previous section, the inverse problem has been expressed as a minimization problem. In order to solve it using a descent algorithm, the gradient of the cost functional should be computed. This is not a straightforward operation: on the one hand due to the implicit dependence of the cost functional with regard to the minimization parameters and on the other hand due to the direct contact problem involving non-differential relations between different fields.

One can ask if the non-differentiability relation due to contact implies a nondifferentiability of the cost functional with respect to the material parameters. Nondifferentiability would certainly occur, if the cost functional is minimized with regards to the parameters of the contact itself as shown in a review paper by Hilding et al [12].

An inspection of some analytical solutions in the classical elastic theory $[7,14]$ shows that the cost functional is differentiable with respect to elastic moduli, the minimization parameters in our case. Numerical computations in a series of configurations involving bimaterial or functional gradient materials exhibit the same conclusion (figure 7).

In the following, we shall propose a gradient computation using the adjoint state method. The adjoint state method permits one to compute the gradient of a constrained cost functional using the solution to an auxiliary problem, called the adjoint problem $[1,2,17]$. This technique will be adapted to the contact problem using the penalized and mixed formulations of the direct contact problem.

\subsection{The adjoint state method based on the penalized formulation $\left(\mathcal{P}_{\epsilon}^{-1}\right)$}

For a small $\epsilon>0$, the inverse problem is expressed as follows:

$$
\begin{aligned}
& \text { minimize } \mathcal{J}_{\epsilon}=\frac{1}{2}\left(F_{\epsilon}-F^{\exp }\right)^{2} \text { with respect to } c \in \boldsymbol{L}, \\
& \text { where } F_{\epsilon} \text { is computed from the solution of }\left(\mathcal{P}_{\epsilon}\right) .
\end{aligned}
$$

As we handle a constrained minimization problem, it is natural to introduce a Lagrangian $\mathcal{L}_{\epsilon}$, by adjoining to the penalized cost functional $\mathcal{J}_{\epsilon}$ the variational equality of the penalized direct problem $\left(\mathcal{P}_{\epsilon}\right)$ :

$\mathcal{L}_{\epsilon}\left(\boldsymbol{u}_{\epsilon}, \boldsymbol{v}_{\epsilon}, \boldsymbol{c}\right)=\frac{1}{2}\left(F_{\epsilon}-F^{\exp }\right)^{2}+\int_{\Omega} \boldsymbol{\sigma}\left(\boldsymbol{u}_{\epsilon}\right): \varepsilon\left(\boldsymbol{v}_{\epsilon}\right) \mathrm{d} \Omega+\frac{1}{2 \epsilon} \int_{\Gamma_{C}}\left\langle u_{\epsilon 2}-g-U^{\exp }\right\rangle_{+}^{2} v_{\epsilon 2} \mathrm{~d} \Gamma$

where $F_{\epsilon}$ is given by (8) and $\left(\boldsymbol{u}_{\epsilon}, \boldsymbol{v}_{\epsilon}, \boldsymbol{c}\right) \in \boldsymbol{V}^{2} \times \boldsymbol{L}$. 
All variables are supposed mutually independent, the virtual displacement field $\boldsymbol{v}_{\epsilon}$ plays the role of a Lagrange multiplier. The construction of the Lagrangian ensures that the saddle point of the $\mathcal{L}_{\epsilon}$ gives the minimum of $\mathcal{J}_{\epsilon}$ and that $\mathcal{L}_{\epsilon} \equiv \mathcal{J}_{\epsilon}$ if $\boldsymbol{u}_{\epsilon}$ is a solution to the direct problem $\left(\mathcal{P}_{\epsilon}\right)$.

The necessary conditions of stationarity of $\mathcal{L}$ can be formally written as

$$
\begin{aligned}
& \left(\frac{\partial \mathcal{L}_{\epsilon}}{\partial \boldsymbol{v}_{\epsilon}}, \boldsymbol{w}\right)=\int_{\Omega} \boldsymbol{\sigma}\left(\boldsymbol{u}_{\epsilon}\right): \varepsilon(\boldsymbol{w}) \mathrm{d} \Omega \\
& +\frac{1}{2 \epsilon} \int_{\Gamma_{C}}\left\langle u_{\epsilon 2}-g-U^{\exp }\right\rangle_{+}^{2} w_{2} \mathrm{~d} \Gamma=0 \quad \forall \boldsymbol{w} \in \boldsymbol{V} \\
& \left(\frac{\partial \mathcal{L}_{\epsilon}}{\partial \boldsymbol{u}_{\epsilon}}, \boldsymbol{w}\right)=\int_{\Omega} \boldsymbol{\sigma}\left(\boldsymbol{v}_{\epsilon}\right): \varepsilon(\boldsymbol{w}) \mathrm{d} \Omega \\
& +\frac{1}{\epsilon} \int_{\Gamma_{C}}\left\langle u_{\epsilon 2}-g-U^{\exp }\right\rangle_{+} w_{2}\left(v_{\epsilon 2}-\left(F_{\epsilon}-F^{\exp }\right)\right) \mathrm{d} \Gamma=0 \quad \forall \boldsymbol{w} \in \boldsymbol{V} \\
& {\left[\frac{\partial \mathcal{L}_{\epsilon}}{\partial \boldsymbol{c}}, \boldsymbol{d}-\boldsymbol{c}\right]=\int_{\Omega} \varepsilon\left(\boldsymbol{u}_{\epsilon}\right): \frac{\partial \boldsymbol{A}}{\partial \boldsymbol{c}}: \varepsilon\left(\boldsymbol{v}_{\epsilon}\right) \cdot(\boldsymbol{d}-\boldsymbol{c}) \mathrm{d} \Omega \geqslant 0 \quad \forall \boldsymbol{d} \in \boldsymbol{L}}
\end{aligned}
$$

where (., .) and [., .] are respectively the duality pairing on $\boldsymbol{V}^{\prime} \times \boldsymbol{V}$ and $\boldsymbol{L}^{\prime} \times \boldsymbol{L}$.

As expected, the derivation of the Lagrangian with respect to the adjoint variable $\boldsymbol{v}_{\epsilon}(16)$ returns the variational formulation of the penalized direct problem. The derivation of the Lagrangian with respect to the direct variable $\boldsymbol{u}_{\epsilon}(17)$ returns the weak formulation of a elastic problem called the penalized adjoint problem $\left(\mathcal{P}_{\epsilon}^{\text {adj }}\right)$.

Let us choose a point $\left(\boldsymbol{u}_{\epsilon}, \boldsymbol{v}_{\epsilon}, \boldsymbol{c}\right)$. If $\boldsymbol{u}_{\epsilon}$ is solution of $\left(\mathcal{P}_{\epsilon}\right)$, by definition of $\mathcal{L}_{\epsilon}$, we have $\mathcal{L}_{\epsilon} \equiv \mathcal{J}_{\epsilon}$. Moreover if $\boldsymbol{v}_{\epsilon}$ is solution of $\left(\mathcal{P}_{\epsilon}^{\text {adj }}\right)$ it follows that

$$
\nabla_{c} \mathcal{J}_{\epsilon}=\frac{\partial \mathcal{L}_{\epsilon}}{\partial \boldsymbol{c}}=\int_{\Omega} \varepsilon\left(\boldsymbol{u}_{\epsilon}\right): \frac{\partial \boldsymbol{A}}{\partial \boldsymbol{c}}: \varepsilon\left(\boldsymbol{v}_{\epsilon}\right) \mathrm{d} \Omega
$$

Thus the explicit expression of the gradient of $\mathcal{J}_{\epsilon}$ has been obtained using the solution to the direct and the adjoint penalized problems.

The previous result has been established within the penalized formulation. It is interesting to know how it evolves as $\epsilon \rightarrow 0$. Without giving a precise mathematical proof, the following results can be conjectured.

- The solution $\boldsymbol{v}_{\epsilon}$ to the penalized adjoint problem $\left(\mathcal{P}_{\epsilon}^{\mathrm{adj}}\right)$ converges to the solution $\boldsymbol{v}$ to the adjoint problem $\left(\mathcal{P}^{\text {adj }}\right)$ driven by the imposed displacements $\left(F-F^{\text {exp }}\right)$ on the effective contact surface $\Gamma_{C}^{\mathrm{eff}}$ :

find $v \in V^{\text {adj }}$ such that

$$
\begin{aligned}
& \int_{\Omega} \boldsymbol{\sigma}(\boldsymbol{v}): \varepsilon(\boldsymbol{w}) \mathrm{d} \Omega=0 \\
& \forall \boldsymbol{w} \in \boldsymbol{V}^{\mathrm{adj}}=\left\{\boldsymbol{w} \in \boldsymbol{V} \mid w_{2}=\left(F-F^{\mathrm{exp}}\right) \text { on } \Gamma_{C}^{\mathrm{eff}}\right\}
\end{aligned}
$$

where $F, \Gamma_{C}$ eff are respectively the force on the indenter and the effective contact surface, computed in the direct contact problem $(\mathcal{P})$.

- The limits and the gradient operations are commutative, i.e.

$$
\lim _{\epsilon \rightarrow 0} \nabla_{c} \mathcal{J}_{\epsilon}=\nabla_{c}\left(\lim _{\epsilon \rightarrow 0} \mathcal{J}_{\epsilon}\right)=\nabla_{c} \mathcal{J} .
$$

As a consequence the gradient of the cost functional can be computed using the following formula:

$$
\nabla_{c} \mathcal{J}=\int_{\Omega} \varepsilon(\boldsymbol{u}): \frac{\partial \boldsymbol{A}}{\partial \boldsymbol{c}}: \varepsilon(\boldsymbol{v}) \mathrm{d} \Omega
$$

where $\boldsymbol{u}$ and $\boldsymbol{v}$ are the solutions to the direct problem $(\mathcal{P})$ and respectively the adjoint problem $\left(\mathcal{P}^{\text {adj }}\right)$. 


\subsection{The adjoint state method based on the mixed formulation $\left(\mathcal{P}_{m}^{-1}\right)$}

This problem is expressed as follows:

$$
\begin{aligned}
& \text { minimize } \mathcal{J}=\frac{1}{2}\left(F-F^{\exp }\right)^{2} \text { with respect to } c \in L, \\
& \text { where } F \text { is computed from the solution to }\left(\mathcal{P}_{m}\right) .
\end{aligned}
$$

The corresponding Lagrangian is expressed as

$$
\begin{aligned}
& \mathcal{L}(\boldsymbol{u}, \boldsymbol{v}, p, q, \boldsymbol{c})=\frac{1}{2}\left(F-F^{\exp }\right)^{2}-\int_{\Omega} \boldsymbol{\sigma}(\boldsymbol{u}): \varepsilon(\boldsymbol{v}) \mathrm{d} \Omega \\
&+\int_{\Gamma_{C}} p \cdot v_{2} \mathrm{~d} \Gamma+\int_{\Gamma_{C}} q \cdot\left(u_{2}-U-g\right) \mathrm{d} \Gamma
\end{aligned}
$$

where $F$ is given by (10), $(\boldsymbol{u}, \boldsymbol{v}, p, q, \boldsymbol{c}) \in \boldsymbol{V}^{2} \times \boldsymbol{Q}^{2} \times \boldsymbol{L}$ and $\boldsymbol{Q}=\left\{q \in\left(H^{1 / 2}\left(\Gamma_{C}\right)\right)^{\prime} \mid q=\right.$ 0 on $\left.\Gamma_{C} / \Gamma_{C}^{\text {eff }}\right\}$.

Let us remark that, opposite to the classical application of the adjoint state method, $q$ has not been sought in the closed convex $N$. If $q$ was in $N$, the stationarity conditions would turn out to be a set of coupled variational inequalities, which are ineffective for practical search of the adjoint state.

With $q \in Q$, the necessary conditions of stationarity of $\mathcal{L}$ can be formally written as follows:

$$
\begin{array}{ll}
\left(\frac{\partial \mathcal{L}}{\partial \boldsymbol{v}}, \boldsymbol{w}\right)=\int_{\Omega} \boldsymbol{\sigma}(\boldsymbol{u}): \varepsilon(\boldsymbol{w}) \mathrm{d} \Omega-\int_{\Gamma_{C}} p \cdot w_{2} \mathrm{~d} \Gamma=0 & \forall \boldsymbol{w} \in \boldsymbol{V} \\
\left(\frac{\partial \mathcal{L}}{\partial \boldsymbol{u}}, \boldsymbol{w}\right)=\int_{\Omega} \boldsymbol{\sigma}(\boldsymbol{v}): \varepsilon(\boldsymbol{w}) \mathrm{d} \Omega-\int_{\Gamma_{C}} q \cdot w_{2} \mathrm{~d} \Gamma=0 & \forall \boldsymbol{w} \in \boldsymbol{V} \\
\left\{\frac{\partial \mathcal{L}}{\partial q}, s\right\}=\int_{\Gamma_{C}} s \cdot\left(u_{2}-U-g\right) \mathrm{d} \Gamma=0 \quad \forall s \in \boldsymbol{Q} & \\
\left\{\frac{\partial \mathcal{L}}{\partial p}, s\right\}=\int_{\Gamma_{C}} s \cdot\left(v_{2}-F^{\exp }+F\right) \mathrm{d} \Gamma=0 \quad \forall s \in \boldsymbol{Q} & \\
{\left[\frac{\partial \mathcal{L}}{\partial \boldsymbol{c}}, \boldsymbol{d}-\boldsymbol{c}\right]=\int_{\Omega} \varepsilon(\boldsymbol{u}): \frac{\partial \boldsymbol{A}}{\partial \boldsymbol{c}}: \boldsymbol{\varepsilon}(\boldsymbol{v}) \cdot(\boldsymbol{d}-\boldsymbol{c}) \mathrm{d} \Omega \geqslant 0} & \forall \boldsymbol{d} \in \boldsymbol{L}
\end{array}
$$

where $\{.,$.$\} denotes the duality pairing on Q^{\prime} \times Q$.

In this case, the derivation of the Lagrangian with respect to the adjoint variables $v$ (24) and $q$ (26) does not return the mixed formulation $\left(\mathcal{P}_{m}\right)$ of the direct problem. Nevertheless, the solutions to $\left(\mathcal{P}_{m}\right)$ are also solutions to (24) and (26).

The derivation of the Lagrangian with respect to the direct variables $\boldsymbol{u}(25)$ and $p$ (27) returns an equivalent formulation of the adjoint problem $\left(\mathcal{P}^{\text {adj }}\right)(20)$.

Therefore if $(\boldsymbol{u}, p)$ is solution to the direct problem $(\mathcal{P})$ and $(\boldsymbol{v}, q)$ is solution to the adjoint problem $\left(\mathcal{P}^{\text {adj }}\right)$, then (24)-(27) are verified. As a consequence, in the penalized case, the gradient can be expressed as

$$
\nabla_{\boldsymbol{c}} \mathcal{J}=\frac{\partial \mathcal{L}}{\partial \boldsymbol{c}}=\int_{\Omega} \varepsilon(\boldsymbol{u}): \frac{\partial \boldsymbol{A}}{\partial \boldsymbol{c}}: \varepsilon(\boldsymbol{v}) \mathrm{d} \Omega
$$

\subsection{Remarks on the adjoint problem $\left(\mathcal{P}^{\mathrm{adj}}\right)$}

For problems with Dirichlet or Neumann boundary conditions described by variational equalities, the application of the adjoint state method leads to a linear adjoint problem described by variational equalities $[1,2,17]$. 


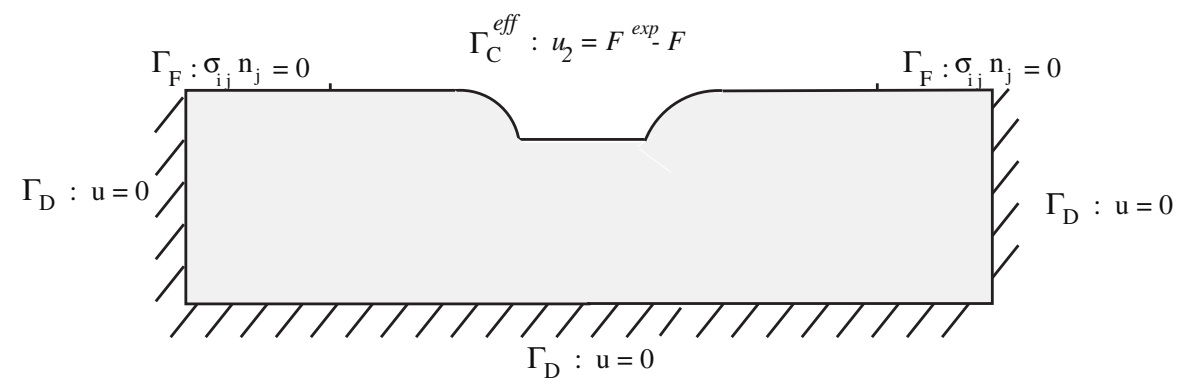

Figure 3. Adjoint problem $\left(\mathcal{P}^{\mathrm{adj}}\right)$.

Table 1. Gradient comparisons between finite difference method (FDM) and adjoint state method (ASM).

\begin{tabular}{rlrr}
\hline$E^{\exp }$ & $\begin{array}{l}E \\
\mathrm{GPa}\end{array}$ & $\begin{array}{c}\nabla \mathcal{J}(E) \\
(\mathrm{ASa})\end{array}$ & \multicolumn{1}{c}{$\begin{array}{r}\nabla \mathcal{J}(E) \\
(\mathrm{FDM})\end{array}$} \\
\hline 200 & 100 & -34.609 & -34.607 \\
200 & 170 & -10.383 & -10.380 \\
200 & 300 & 34.609 & 34.614 \\
200 & 230 & 10.383 & 10.387 \\
20 & 10 & -3.461 & -3.461 \\
20 & 30 & 3.461 & 3.461 \\
\hline
\end{tabular}

It is important to point out that, for problems described by variational inequalities, the obtained adjoint problem $\left(\mathcal{P}^{\text {adj }}\right)$ is linear with Dirichlet boundary conditions (see figure 3 ), and therefore described by a variational equality (20).

From a practical point of view, this implies that the overburden of computing the adjoint problem, and implicitly the gradient of the cost functional, is very small.

\section{Numerical examples}

The gradient expressions obtained previously have been applied in a series of numerical examples:

- comparison of gradient evaluations of the cost functional $\mathcal{J}$ using the adjoint state method (ASM) and the finite difference method (FDM) (see table 1).

- identification of the elastic moduli of a bimaterial

- identification of the elastic moduli of a functional gradient material with Young's moduli depending linearly on radius or depth.

For the identification problems, all indentation experiments have been simulated by direct computations.

The direct and the adjoint problems have been programmed in the object oriented language gibiane of the finite element code CASTEM 2000 [3]. From a numerical point of view the contact conditions have been treated in an exact manner, i.e. without use of penalization. Fournoded linear quadrangular elements have been employed and a typical mesh is presented in figure 4.

As an indenter, spheres of different radiuses and cones with different semi-angles have been used without changing the quality of the final results. 
Figure 4. An axisymmetric mesh used for identification simulation.

Computations have been performed on HP 9000 workstation, on which a contact computation for the mesh shown took $30 \mathrm{~s}$ CPU time.

In the next examples, two parameters $\left(c_{1}, c_{2}\right)$ characterizing the material were identified from two pairs of displacement-force measurements from the identation curve (see figure 1). Therefore two cost functionals were defined as follows:

$$
\mathcal{J}_{1}\left(c_{1}, c_{2}\right)=\frac{1}{2}\left(F-F_{1}^{\text {exp }}\right)^{2} \quad \mathcal{J}_{2}\left(c_{1}, c_{2}\right)=\frac{1}{2}\left(F-F_{2}^{\exp }\right)^{2} .
$$

The relaxation algorithm used can be described as follows:

(i) Choose a initial pair $\left(c_{1}, c_{2}\right)$ and two identification precisions $\varepsilon_{1}>0, \varepsilon_{2}>0$

(ii) While $\mathcal{J}_{1} \geqslant \varepsilon_{1}$ or $\mathcal{J}_{2} \geqslant \varepsilon_{1}$ :

(a) minimize $\mathcal{J}_{1}$ with respect to $c_{1}$, until $\frac{\partial \mathcal{J}_{1}}{\partial c_{1}}<\varepsilon_{2}$

(b) minimize $\mathcal{J}_{2}$ with respect to $c_{2}$, until $\frac{\partial \mathcal{J}_{2}}{\partial c_{2}}<\varepsilon_{2}$

where the minimization is performed using a Newton method.

This method proved to be more efficient than minimizing directly $\mathcal{J}_{1}, \mathcal{J}_{2}$, or $\mathcal{J}_{1}+\mathcal{J}_{2}$ with respect to both $E_{1}$ and $E_{2}$ using a BFGS method [6].

\subsection{The bimaterial}

Identifications have been performed in the case of a cylinder composed of two perfectly bonded elastic coatings (figure 5). The thickness of the coating has been considered as a priori known and only the values of the two Young's moduli, i.e. $c=\left(E_{1}, E_{2}\right)$, have been identified from simulated measurements.

The two values choosen from the complete indentation curve $\left(U^{\exp }(t), F^{\exp }(t)\right)$, correspond to indentation depths smaller and respectively larger than the coating $\left(U_{1}^{\text {exp }}=\right.$ $0.2 \mathrm{~mm}$ and $U_{2}^{\exp }=2.0 \mathrm{~mm}$ ).

From a mechanical point of view, this means that the load will be carried in different proportions by the coating and the substrate. This can be observed from the shape of the cost functionals $\mathcal{J}_{1}\left(E_{1}, E_{2}\right)$ and $\mathcal{J}_{2}\left(E_{1}, E_{2}\right)$ : in figure 7 , we observe that both cost functionals present a narrow valley and that $\mathcal{J}_{1}$ is essentially sensitive to variations of $E_{1}$ (substrate) and that $\mathcal{J}_{2}$ is essentially sensitive to variations of $E_{2}$ (coating).

In all tested cases, the identification converges after some 30 iterations (figure 6) with identified moduli within $0.001 \%$ of the experimental values. A series of results corresponding to different starting points are presented in table 2. 


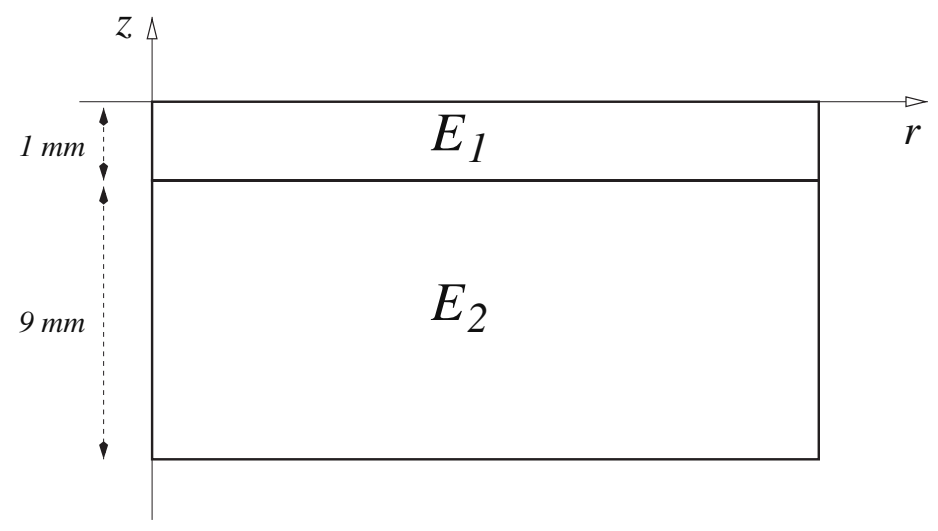

Figure 5. Bimaterial used for the identification.

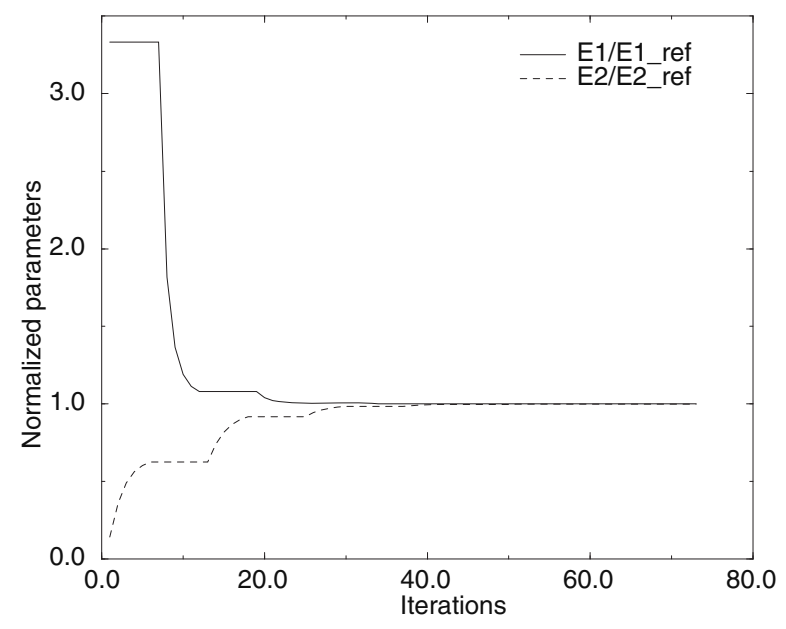

Figure 6. Convergence of the normalized elastic coefficients for the bimaterial.

Table 2. Identification results for the bimaterial.

\begin{tabular}{lll}
\hline $\begin{array}{l}\text { Starting } \\
\left(E_{1}, E_{2}\right)\end{array}$ & $\begin{array}{l}\text { Final } \\
\left(E_{1}, E_{2}\right)\end{array}$ & $\begin{array}{l}\text { Reference } \\
\left(E_{1}, E_{2}\right) \\
\left(10^{5} \mathrm{MPa}\right)\end{array}$ \\
\hline$\left(10^{5} \mathrm{MPa}\right)$ & $\left(10^{5} \mathrm{MPa}\right)$ \\
\hline$(0.4,0.4)$ & $(1.200,2.801)$ & $(1.200,2.800)$ \\
$(0.4,4.0)$ & $(1.200,2.801)$ & $(1.200,2.800)$ \\
$(4.0,0.4)$ & $(1.200,2.799)$ & $(1.200,2.800)$ \\
$(4.0,4.0)$ & $(1.200,2.799)$ & $(1.200,2.800)$ \\
\hline
\end{tabular}

\subsection{The functional gradient materials}

Identifications have been performed for a cylinder with Young's modulus depending on depth $\left(E(r, z)=E_{1} z+E_{2}\right)$ or on radius $\left(E(r, z)=E_{1} r+E_{2}\right)$.

The previous identification strategy has been used for these problems.

In all cases, the identification converges after some 30 iterations with identified moduli within $0.001 \%$ of the experimental values. Identification results corresponding to different starting points are show in tables 3 and 4 .

In the case of a Young's modulus depending on depth $z$, convergence required more 

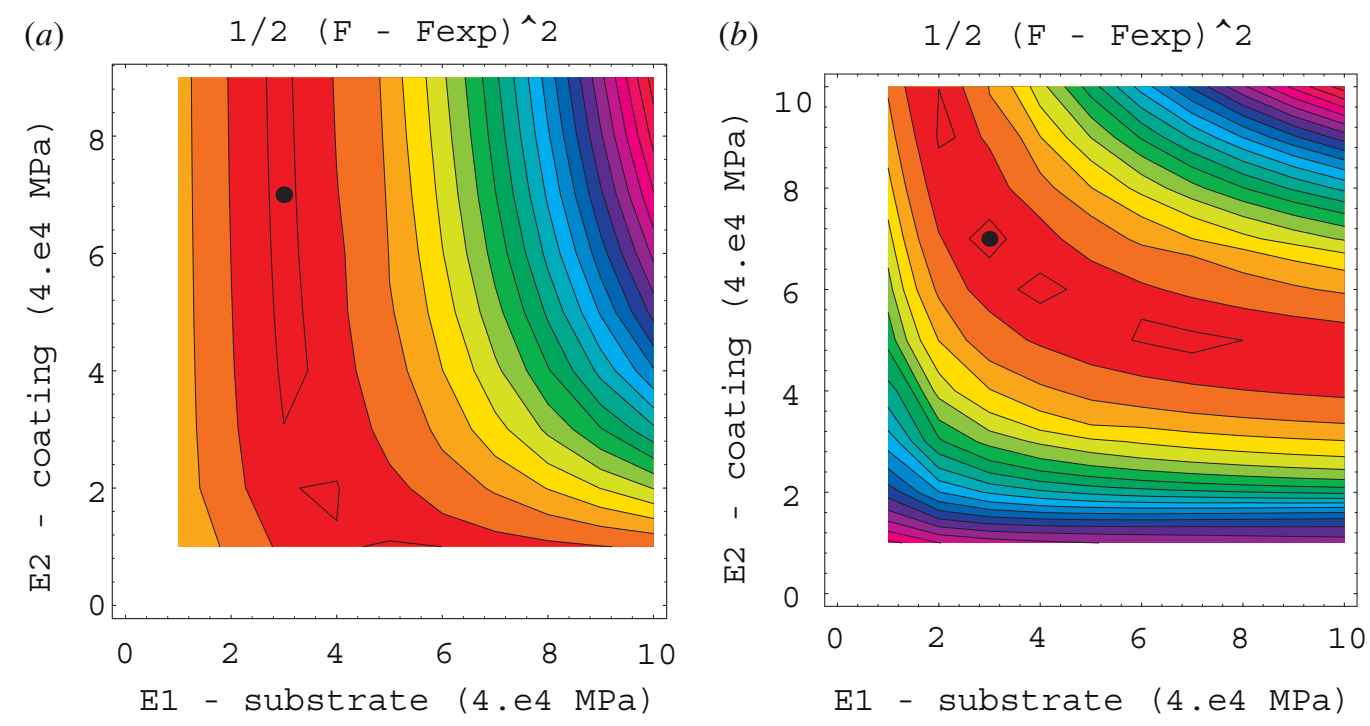

Figure 7. Contourplots of $\mathcal{J}_{1}$ for a deep $\left(U^{\exp }=0.2 \mathrm{~mm}\right)$ and $\mathcal{J}_{2}$ for a small indentation $\left(U^{\exp }=\right.$ $2.0 \mathrm{~mm})$.

Table 3. Identification results for the radius functional gradient material.

\begin{tabular}{lll}
\hline $\begin{array}{l}\text { Starting } \\
\left(E_{1}, E_{2}\right)\end{array}$ & $\begin{array}{l}\text { Final } \\
\left(E_{1}, E_{2}\right)\end{array}$ & $\begin{array}{l}\text { Reference } \\
\left(E_{1}, E_{2}\right)\end{array}$ \\
$\left.10^{5}(\mathrm{MPa} \mathrm{m})^{-1}, \mathrm{MPa}\right)$ & $10^{5}\left(\mathrm{MPa} \mathrm{m}^{-1}, \mathrm{MPa}\right)$ & $10^{5}\left(\mathrm{MPa} \mathrm{m}^{-1}, \mathrm{MPa}\right)$ \\
\hline$(0.4,0.4)$ & $(1.200,2.801)$ & $(1.200,2.800)$ \\
$(0.4,4.0)$ & $(1.200,2.801)$ & $(1.200,2.800)$ \\
$(4.0,0.4)$ & $(1.201,2.798)$ & $(1.200,2.800)$ \\
$(4.0,4.0)$ & $(1.200,2.799)$ & $(1.200,2.800)$ \\
\hline
\end{tabular}

Table 4. Identification results for the depth functional gradient material.

\begin{tabular}{lll}
\hline $\begin{array}{l}\text { Starting } \\
\left(E_{1}, E_{2}\right)\end{array}$ & $\begin{array}{l}\text { Final } \\
\left(E_{1}, E_{2}\right)\end{array}$ & $\begin{array}{l}\text { Reference } \\
\left(E_{1}, E_{2}\right)\end{array}$ \\
$10^{5}\left(\mathrm{MPa} \mathrm{m}^{-1}, \mathrm{MPa}\right)$ & $10^{5}\left(\mathrm{MPa} \mathrm{m}^{-1}, \mathrm{MPa}\right)$ & $10^{5}\left(\mathrm{MPa} \mathrm{m}^{-1}, \mathrm{MPa}\right)$ \\
\hline$(0.4,0.4)$ & $(1.199,2.805)$ & $(1.200,2.800)$ \\
$(0.4,4.0)$ & $(1.199,2.806)$ & $(1.200,2.800)$ \\
$(4.0,0.4)$ & $(1.201,2.791)$ & $(1.200,2.800)$ \\
$(4.0,4.0)$ & $(1.200,2.797)$ & $(1.200,2.800)$ \\
\hline
\end{tabular}

iterations than in the radius dependency case (50 iterations) and results were slightly less accurate. This can be explained by the important radial strain under a blunt conical indenter $[7,14]$.

\section{Conclusion}

This paper presented the application of the adjoint state method to variational inequalities, through the identification of elastic coefficients from an indentation test. Using two different reasonements, based on the mixed and penalized formulations of the contact Signorini problem, it has been shown that the adjoint problem is completely linear with Dirichlet boundary 
conditions on the effective contact area. The effective contact area has been previously detected in the direct problem.

The effectiveness of the method is illustrated on numerical examples using the finite element method. An extension of the method to evolution equations, corresponding to nonlinear constitutive laws and large strains is also possible. An application for viscoplastic materials is currently under development $[4,19]$.

In spite of promising numerical results, the presented method needs a precise mathematical proof. Other fundamental questions related to the indentation identification problem, such as uniqueness, stability, choice of a convex cost functional etc, are also open problems.

\section{Acknowledgments}

The authors would like to thank Huy Duong Bui, Stephane Andrieux, Laurent Bourgeois and Said Taheri for helpful discussions during the fullfilment of this work.

\section{References}

[1] Bourgeois L 1998 Contrôle optimal et problèmes inverses en plasticité PhD Thesis Ecole Polytechnique, France

[2] Bui H D 1993 Introduction aux Problèmes Inverses en Mécanique des Matériaux (Paris: Eyrolles) (Engl. Transl. (Boca Raton, FL: CRC Press))

[3] Castem2000 Finite Element Code webpage http://www.castem.org

[4] Constantinescu A and Tardieu N 1999 On the identification of elastoviscoplastic constitutive laws from indentation tests Inverse Problems in Engineering at press

[5] Doener M F and Nix W D 1986 A method of interpreting the data from depth-sensing indentation measurements J. Mater. Res. 1 601-9

[6] Gill P E, Murray W and Wright M H 1981 Practical Optimization (New York: Academic)

[7] Gladwell G M L 1980 Contact Problems in the Classical Theory of Elasticity (Sijthoff and Noordhoff)

[8] Hainsworth S V, Chandler H W and Page T F 1996 Analysis of nanoindentation load-displacement loading curves J. Mater. Res. 11 1987-95

[9] Hasanov A and Seyidmamedov Z 1995 The solution of an axisymmetric inverse elasto-plastic problem using penetration diagrams Int. J. Non-Linear Mech. 30 465-77

[10] Hasanov A 1997 Inverse coefficient problems for monotone potentials operators Inverse Problems 13 1265-78

[11] Hasanov A 1998 Inverse coefficient problems for elliptic variational inequalities with a nonlinear monotone operator Inverse Problems 14 1151-70

[12] Hilding D, Klarbring A and Petersson J 1999 Optimization of structures in unilateral contact ASME Appl. Mech. Rev. 52 139-57

[13] Hill R, Lee E H and Tupper S J 1947 The theory of wedge indentation of ductile materials Proc. R. Soc. A 188 273-90

[14] Johnson K L 1985 Contact Mechanics (Cambridge: Cambridge University Press)

[15] Kikuchi N and Oden J T 1988 Contact Problems in Elasticity: a Study of Variational Inequalities and Finite Element Methods (Philadelphia: SIAM)

[16] Koguchi H 1999 Determination of mechanical properties of thin films and functional gradient materials using an inverse technique Conf. on Inverse Problems in Engineering: Theory and Practice (ASME)

[17] Lions J L 1968 Contrôle Optimal des Systèmes Gouvernés par des Équations aux Dérivées Partielles (Dunod)

[18] Tabor D 1951 Hardness of Metals (Oxford: Oxford University Press)

[19] Tardieu N and Constantinescu A 1999 On the identification of nonlinear constitutive laws from indentation tests Conf. on Inverse Problems in Engineering: Theory and Practice (ASME) 